IPMU-08-0034

\title{
Electron and Photon Energy Deposition in Universe
}

\author{
Toru Kanzaki ${ }^{1}$, Masahiro Kawasaki ${ }^{1,2}$ \\ ${ }^{1}$ Institute for Cosmic Ray Research, University of Tokyo, Kashiwa 277-8582, JAPAN \\ ${ }^{2}$ Institute for the Physics and Mathematics of the Universe, University of Tokyo, \\ Kashiwa 277-8582, JAPAN
}

\begin{abstract}
.
We consider energy deposition of high energy electrons and photons in universe. We carry out detailed calculations of fractions of the initial energy of the injected electron or photon which are used to heat, ionize and excite background plasma in the early universe for various ionization states and redshifts.
\end{abstract}

\section{Introduction}

The energy deposition of fast electrons and high energy photons in partly ionized plasma is an important issue in the wide range of physics and astrophysics. In cosmology, for example, the high energy photons and/or electrons are injected from decay or annihilation of massive particles. The recent observation of WMAP on Cosmic Microwave Background (CMB) has shown that dark matter constitutes about $80 \%$ of the total mass in the universe [3]. One of the most promising candidates for dark matter is a supersymmetric particle with mass $O(100) \mathrm{GeV}$. If such massive particles are annhilate and/or decay, the ejected charged particles and photons interact with various background particles: electron, atomic hydrogen and CMB photon, and hence gives the significant effects on the thermal histrory of the universe. In this case, to estimate the cosmological effect of dark matter annihilation/decay precisely, we need to understand development of electromagnetic cascade showers induced by primary particles, energy loss of charged particles and photons, and so on. Besides dark matter, there are many candidates for inducing electromagnetic showers and affect the cosmic background plasma in physics beyond the standard model.

Detailed calculations for electrons and photons slowing down in partially ionized plasma of atomic hydrogen were carried out by many authors (see references in [1]). However, these authors mainly studied the case of injected electron energy up to keV. In this paper, we extended these calculations which are valid up to much higher energy, i.e., $1 \mathrm{TeV}$. The injected high energy particles produce the cascade showers and lose their energy. We can categorize three types of energy loss: heat, excitation and ionization according to what the energy of the particle is used for. It is the aim of this paper to derive these quantities precisely. They depend on the energy of primary particles as well 
as redshift and state of ionization. There are two reasons why it is difficult to derive them. One is the large number of interactions involved with the energy degradation. The other is connection between the energy degradation of charged particles and that of photons. For example, high energy electrons can produce photons as much the same energy through inverse Compton scatterings with CMB photons. In other words, we can not calculate the evolution of the energy of charged particles and photons separately but simultaneously.

In section 2, we show the numerical method to calculate the energy degradation of the primary particles. In section 3 , the evolutions of the energy of primary electron, photon and positron are presented. The results of the energy degradation of the primary particles is shown in section 4. In section 5, we summarize the results.

\section{Numerical Method}

For incident electrons and photons, there are many interactions which contribute to energy degradation. It is convenient to divide these processes into two groups. One is the group characterized by losing only a very small portion of energy in one collision (continuous loss). The other is the group characterized by being likely to lose a significant portion of energy in one collision ('catastrophic' loss [6]). In the case of the latter, it is necessary to use an integro-differential equation to calculate electron energy spectrum.

Let $E_{1}, E_{2}, \cdots, E_{N}$ be a discrete set of energies of particles $\left(E_{i}<E_{j}\right.$ for $\left.i<j\right)$ and $N_{p}\left(E_{s}\right) \Delta E_{s}$ be the number of particles with energy between $E_{s}-\Delta E_{s} / 2$ and $E_{s}+\Delta E_{s} / 2$. The accuracy of the numerical method is limited by the bin size $\left(\Delta E_{i}\right)$. Since we consider very large energy region ranges from $10 \mathrm{eV}$ to $100 \mathrm{GeV}$, the bin sizes are taken to increase as energy so that $\Delta E_{i} / E_{i}$ become constant. The particle energy spectrum is given by

$$
\begin{aligned}
\frac{\partial N_{p}\left(E_{s}\right)}{\partial t} & =-\frac{1}{\Delta E_{s}}\left[\frac{-d E}{d t}\right] N_{p}\left(E_{s}\right)-N_{p}\left(E_{s}\right) \sum_{i<s} P\left(E_{s}, E_{i}\right) \\
& +\sum_{i>s} N_{p}\left(E_{i}\right) P\left(E_{i}, E_{s}\right)+Q\left(E_{s}\right),
\end{aligned}
$$

where $P\left(E_{i}, E_{j}\right) d t$ is the probability that a particle with energy $E_{i}$ undergo a collision causing it to lose energy and have energy $E_{j}$ in time $d t$, and $Q\left(E_{i}\right)$ represents sources and sinks of particles corresponding to possible production, annihilation or gradual leakage from the energy range that we consider [4]. The first term of the R.H.S. of Eq. (1) represents continuous loss and the second and third terms represent outflow and inflow caused by catastrophic loss.

In this paper, we are mainly interested in how much initial particle energy convert to heat, excitation and ionization. For convenience, we define $\chi_{h}(E), \chi_{e}(E)$ and $\chi_{i}(E)$ as fractions of the initial energy $E$ which go to heat, excitation and ionization, repectively

$\ddagger$ We frequently use "fraction of heat", "fraction of excitation" and "fraction of ionization" to refer $\chi_{h}$, $\chi_{e}$ and $\chi_{i}$, respectively. Please do not confuse $\chi_{i}$ with "ionization fraction" $x_{e}$ which is the fraction of ionized hydrogens. 
Suppose that we know already $\chi_{h}\left(E_{i}\right), \chi_{e}\left(E_{i}\right)$ and $\chi_{i}\left(E_{i}\right)$ with energy less than $E_{s}$ and consider a particle with initial energy $E_{s}$. Since there are no particle with energy more than $E_{s}$, the energy degradation is characterized by the first term of the R.H.S. of Eq. (1) (continuous loss), the second term (catastrophic loss) and the last term (annihilation). Here we only consider that annihilation of the primary particle contributes to $Q\left(E_{s}\right)$. All these terms are in proportion to $N_{p}\left(E_{s}\right)$, so we define the following number loss function

$$
L\left(E_{s}\right)=\frac{1}{\Delta E_{s}}\left[\frac{-d E}{d t}\right]+\sum_{i} n_{t} v_{p} \sigma_{i}\left(E_{s}\right)
$$

where $n_{t}$ is the number density of target particle, $v_{p}$ is the particle velocity and $\sigma_{i}\left(E_{s}\right)$ is the catastrophic and annihilation cross sections. From number loss function, we can obtain the probability that a particle undergoes a particular collision " $m$ " [5]. The collision frequency for a particular continuous loss may be defined by

$$
\nu_{m}\left(E_{s}, E_{s-1}\right)=\frac{1}{\Delta E_{s}}\left[\frac{-d E}{d t}\right]_{m}
$$

and the collision frequency for a particular catastrophic loss " $\alpha$ " and annihilation " $\beta$ " are given by

$$
\begin{aligned}
& \nu_{\alpha}\left(E_{s}, E_{i}\right)=n_{t} v_{p} \sigma_{\alpha}\left(E_{s}, E_{i}\right), \\
& \nu_{\beta}\left(E_{s}\right)=n_{t} v_{p} \sigma_{\beta}\left(E_{s}\right) .
\end{aligned}
$$

The total collision frequency for the catastrophic loss is given by

$$
\nu_{\alpha}\left(E_{s}\right)=\int d E n_{t} v_{p} \sigma_{\alpha}\left(E_{s}, E\right)
$$

Then the probability $P\left(E_{s}, E_{i}\right)$ is written as

$$
\begin{aligned}
& P\left(E_{s}, E_{i}\right)=\frac{\sum_{\alpha} \nu_{\alpha}\left(E_{s}, E_{i}\right)}{\sum_{m} \nu_{m}\left(E_{s}, E_{s-1}\right)+\sum_{\alpha}\left(\nu_{\alpha}\left(E_{s}\right)+\nu_{\beta}\left(E_{s}\right)\right)}(i \neq s-1), \\
& P\left(E_{s}, E_{s-1}\right)=\frac{\sum_{m} \nu_{m}\left(E_{s}, E_{s-1}\right)+\sum_{\alpha} \nu_{\alpha}\left(E_{s}, E_{s-1}\right)}{\sum_{m} \nu_{m}\left(E_{s}, E_{s-1}\right)+\sum_{\alpha}\left(\nu_{\alpha}\left(E_{s}\right)+\nu_{\beta}\left(E_{s}\right)\right)} .
\end{aligned}
$$

Combining these probabilities with the data about $\chi_{h}(E), \chi_{e}(E)$ and $\chi_{i}(E)$ with energy less than $E_{s}, \chi_{h}\left(E_{s}\right), \chi_{e}\left(E_{s}\right)$ and $\chi_{i}\left(E_{s}\right)$ can be obtained. Please notice that the definition of the frequency for continuous loss depends on the size of the energy bin but final result is independent of it. The reason is as follows. When the all bins are divided into halves, the probability $P\left(E_{s}, E_{i}\right)$ decreases by fifty percent if the bin size is small enough. This is because the collision frequency for continuous loss is much larger than that for catastrophic loss in this case. However, the effect of the decrease of the probability is cancelled by the increase of the bin numbers. We have checked that our results are independent of the size of the energy bin. 


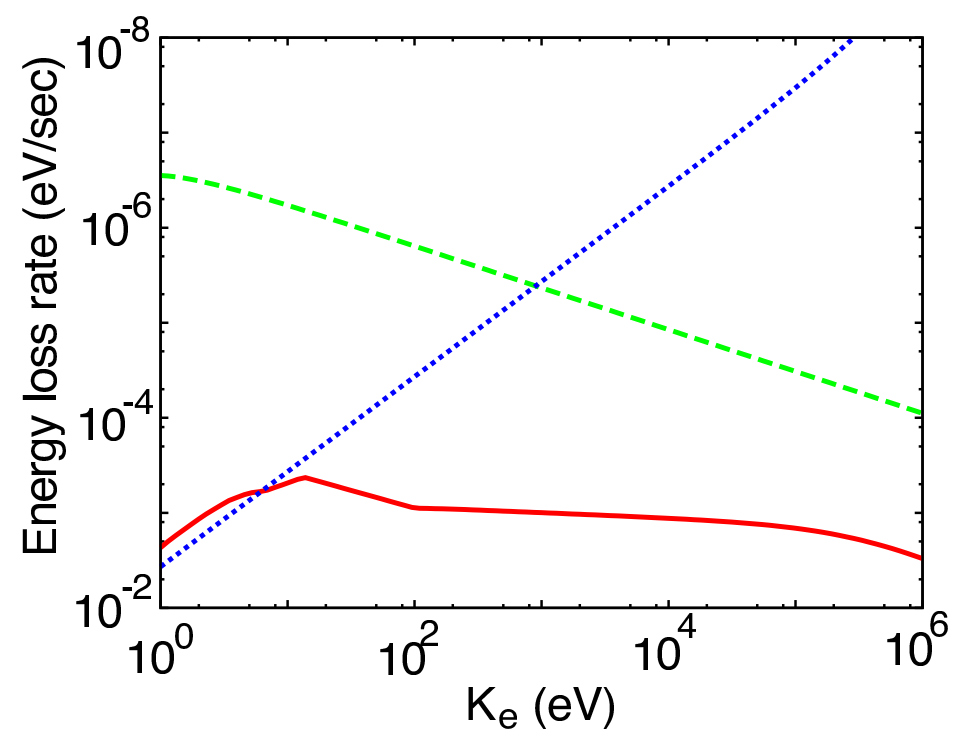

Figure 1. Continuous energy loss rates for electrons with $x_{e}=10^{-2}$ and $1+z=1000$. Solid line represents elastic collision with atomic hydrogen, dashed line represents Coulomb loss with background electron and dotted line represents inverse Compton scattering with CMB photon.

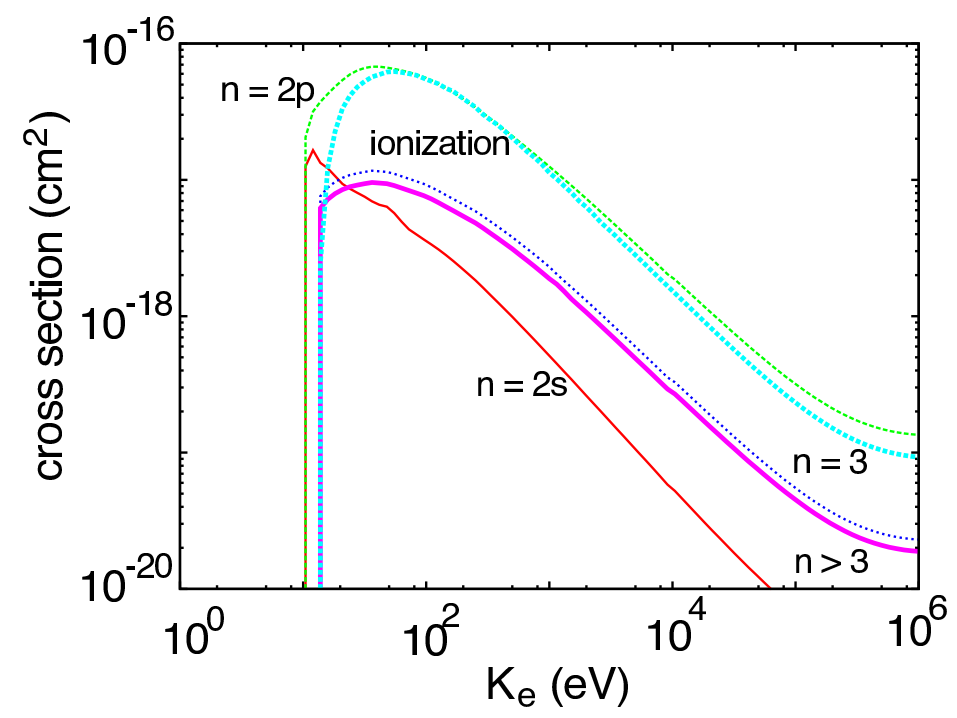

Figure 2. Cross sections for electron impact excitation and ionization of $\mathrm{H}$.

\section{Individual Evolution}

\subsection{Electron}

For incident high energy electrons, there are many interactions which contribute to energy degradation: elastic collision with atomic hydrogen, excitation and ionization of atomic hydrogen, Coulomb loss with background electrons and inverse Compton scattering with background photons (CMB photons). The cross sections and energy 


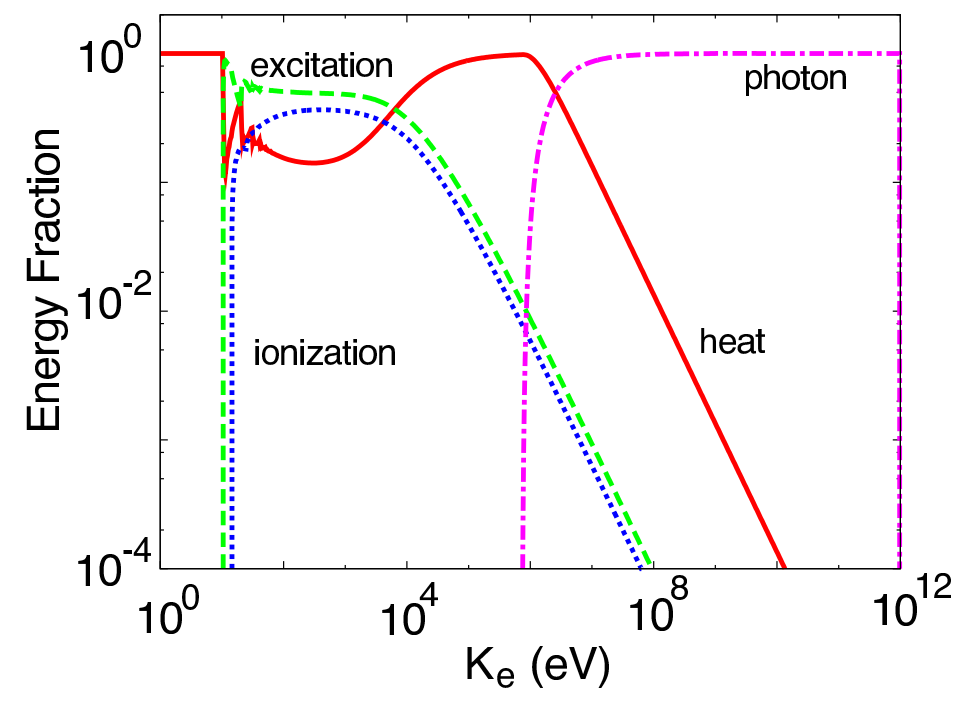

Figure 3. Electron energy degradation with $x_{e}=0$ and $1+z=1000$. Solid line represents $\chi_{h}$, dashed line represents $\chi_{e x}$ and dotted line represents $\chi_{i}$. Dot dashed line represents the fraction of photon energy which energy is larger than Ry.

loss rates of these interactions are described in Appendix A and shown Figs. 1 and 2. Hereafter, we use electron kinetic energy $\left(K_{e}\right)$ instead of electron energy $\left(E_{e}=K_{e}+m_{e}\right)$ for convenience. In this paper, we have used 4 level $(2 \mathrm{~s}, 2 \mathrm{p}, n=3$ and $n>3)$ approximation in considering electron impact excitation. From Fig. 1 it is found that energy loss is dominated by collisions with background electron (Coulomb loss) at low energy and inverse Compton scattering off CMB photon at high energy. The reason is simple. At low energy, the average energy loss of electron is roughly $\Delta E_{e} \propto \beta^{2} E_{C M B}$ for one inverse Compton scattering. So electrons lose very few fraction of their energy and hence energy loss is dominated by collisions with atomic hydrogen and background electron. On the other hand, at high energy, since the number density of CMB photon is much larger than that of background electron, energy loss is dominated by inverse Compton scattering. Energy loss by Coulomb collisions is so efficient that free electrons with ionization fraction, $x_{e}$, exceeding $10^{-4}$ have a substantial influence on the energy degradation [1. Thus, as the ionization fraction increases, the fraction of the initial electron energy which is converted to heat $\left(\chi_{h}(E)\right)$ increases as shown later.

We calculate the energy degradation of electron following the method described in the previous section. There are two free parameters: ionization fraction $x_{e}$ and redshift $1+z$. Ionization fraction is relevant for collisions with atomic hydrogens and background electrons, and redshift is mostly relevant for inverse Compton scattering. For simplicity, we regard the distribution of CMB photons as monoenergetic $\left(E_{\gamma}=6.34 \times 10^{-13}(1+z)\right.$ $\mathrm{GeV}$ ), not blackbody. Free and bound proton number densities can be parameterized by baryon-to-photon ratio $\eta: n_{p}=\eta n_{C M B}$. We adopt $\eta=6.1 \times 10^{-10}$ from the result of WMAP 3-year observation [3]. We take into account properly secondary electrons which is produced by electron impact ionization. 


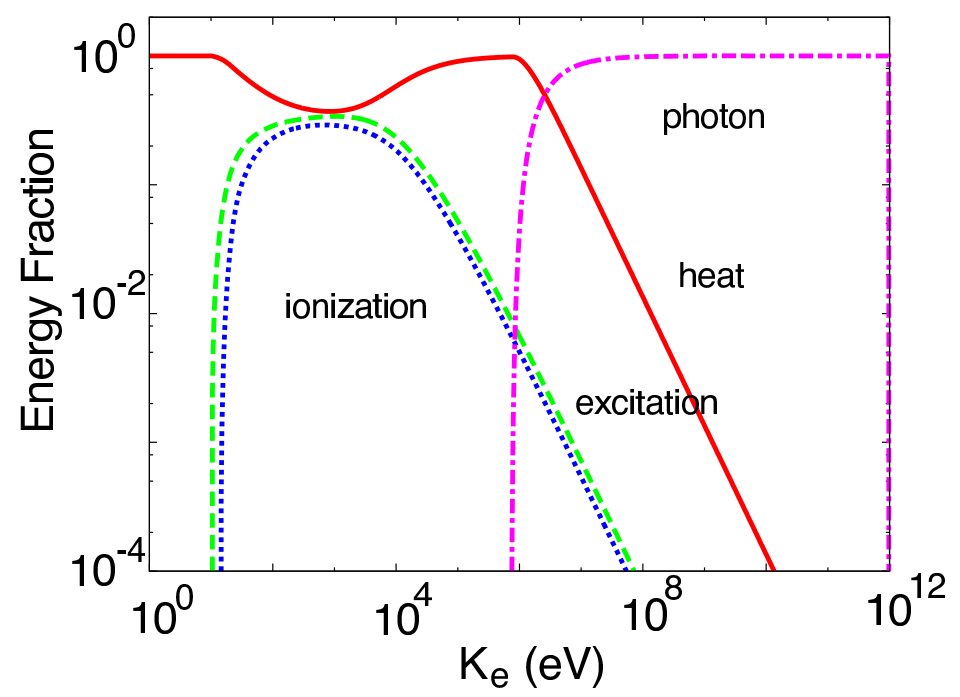

Figure 4. Electron energy degradation with $x_{e}=10^{-2}$ and $1+z=1000$. Solid line represents $\chi_{h}$, dashed line represents $\chi_{e x}$ and dotted line represents $\chi_{i}$. Dot dashed line represents the fraction of photon energy which energy is larger than Ry.

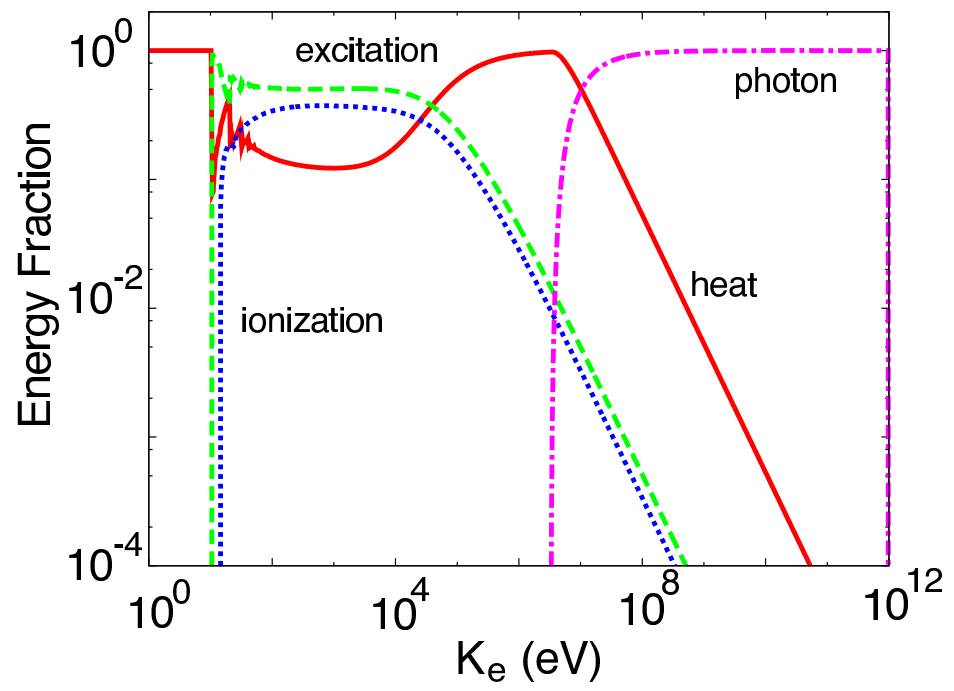

Figure 5. Electron energy degradation with $x_{e}=0$ and $1+z=100$. Solid line represents $\chi_{h}$, dashed line represents $\chi_{e x}$ and dotted line represents $\chi_{i}$. Dot dashed line represents the fraction of photon energy which energy is larger than Ry.

Energy degradation for several values of $x_{e}$ and $1+z$ is shown in Figs. 3 35 , Here, we plot $\chi_{h}(E), \chi_{e x}(E)$ and $\chi_{i}(E)$. In addition, we also plot the fraction of the initial energy that goes to photons with energy larger than $\mathrm{Ry}(=13.6 \mathrm{eV})$. This energy is the threshold energy for photoionization, and it is granted that these photons also contribute to heat, excitation and ionization and we will treat them in Section 4. On the other hnad, photons with energy less than Ry only heat background electrons with Compton scattering. An oscillating behavior below $50 \mathrm{eV}$ in Figs. 3 and 5 reflects the nature of discrete energy loss. This behavior is not seen in Fig. 4 since the effect 
of Coulomb loss dominates over ionization and excitation. The common features in Figs. 3,5 are as follows: (1) the fractions of heat, excitation and ionization are the same order for $E_{e}<10^{4} \mathrm{eV}$, (2) heat dominates at $10^{4}<E_{e}<10^{6} \mathrm{eV}$, and (3) finally the energy of photons dominates for $E_{e}>10^{7} \mathrm{eV}$. At relatively low energy, inverse Compton scattering is inefficient. Moreover, the cross sections and energy loss rates for momentum loss, exitation and ionization are almost the same order if ionization fraction is not large, i.e. Coulomb loss is not dominant. As a consequence, $\chi_{h}, \chi_{e x}$ and $\chi_{i}$ are nearly the same order and our calculation corresponds to the result of [1] up to $E_{e} \sim \mathrm{keV}$. As electron energy increases, inverse Compton scattering becomes significant. If the energy of scattered-up photon is less than the threshold energy of photoionization, the energy loss due to inverse Compton scattering converts to heat and the fraction of heat approaches to unity. When electron energy further increases and most of scattered-up photons have enough energy to ionize atomic hydrogen, the energy of incident electron exclusively converts to the photon energy.

Next we will show how these fractions are dependent on parameters. To see the effect of ionization fraction, let us compare Fig. 3 with Fig. 4. It can be seen that the fraction of heat increases at low energy as ionization fraction increases. This is because Coulomb collision converts initial electron energy into heat exclusively. Besides, the collision frequencies for ionization and excitation are proportional to the number density of hydrogen atom, $\left(1-x_{e}\right)$, and hence they are alomost independent of $x_{e}$ if $x_{e} \ll 1$. Thus, $\chi_{e x}(E)$ and $\chi_{i}(E)$ is smaller as ionization fraction increases. At high energy, the results are irrelevant for ionization fraction since inverse Compton scattering dominates over all other processes. Comparing Fig. 3 with Fig. 5 , it can be seen that the rise of heat and photon energy shifts to high energy side as redshift decreases. This is because the effect of inverse Compton increases in proportion to the energy of CMB photon, and hence in inverse proportion to redshift.

\subsection{Photon}

As well as electron, there are many interactions which contribute to energy degradation for incident high energy photons: photoionization, Compton scattering with background electrons, pair production in matter, photon-photon scattering and double photon pair creation. Here, we neglect photo-excitation since the resultant excited state immediately emits photon and goes down to the ground state. The cross sections and energy loss rates of these interactions are found in Appendix A and shown in Fig. 6.

Energy degradation is plotted for $x_{e}=0$ and $x_{e}=0.98$ in Fig. 7 and Fig. 8 , Here, we plot $\chi_{h}$ and $\chi_{i}$. In addition, we also plot the fraction of initial energy which goes to electrons with energy larger than $0.75 \mathrm{Ry}$. This energy is the threshold for electron impact excitation of atomic hydrogen. Electrons with lower energy only heat background electrons through elastic collision with atomic hydrogen and Coulomb loss. The sudden falls of $\chi_{h}$ reflects the nature of discrete energy loss. The energy for the first fall $\left(E_{\gamma}=\mathrm{Ry}\right)$ corresponds to the threshold energy of photoionization and the second 


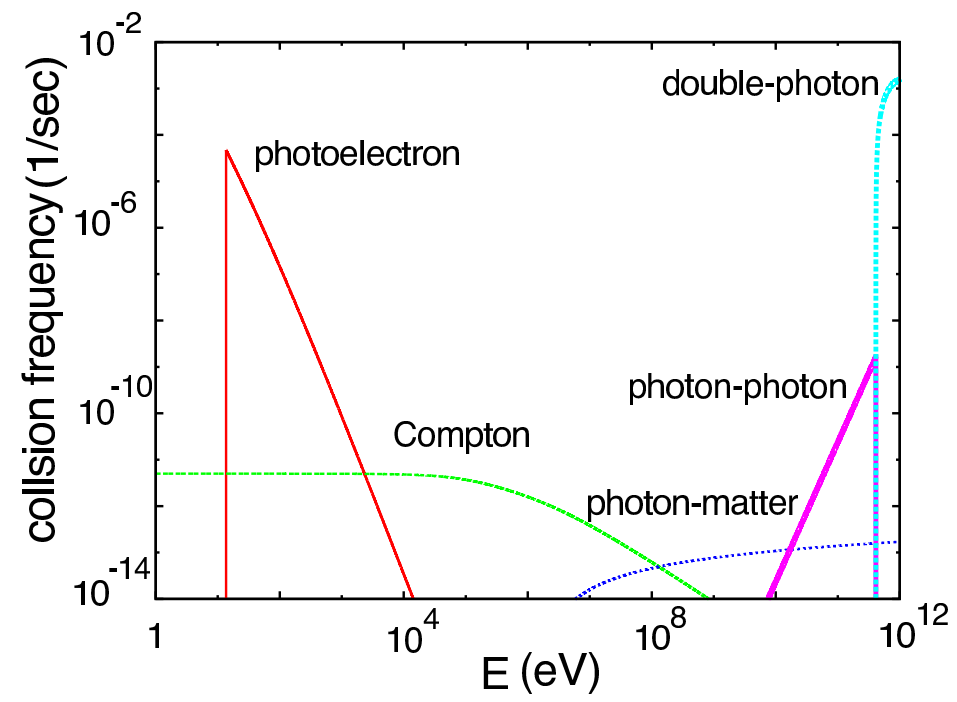

Figure 6. Photon collision frequencies with $x_{e}=0$ and $1+z=1000$.

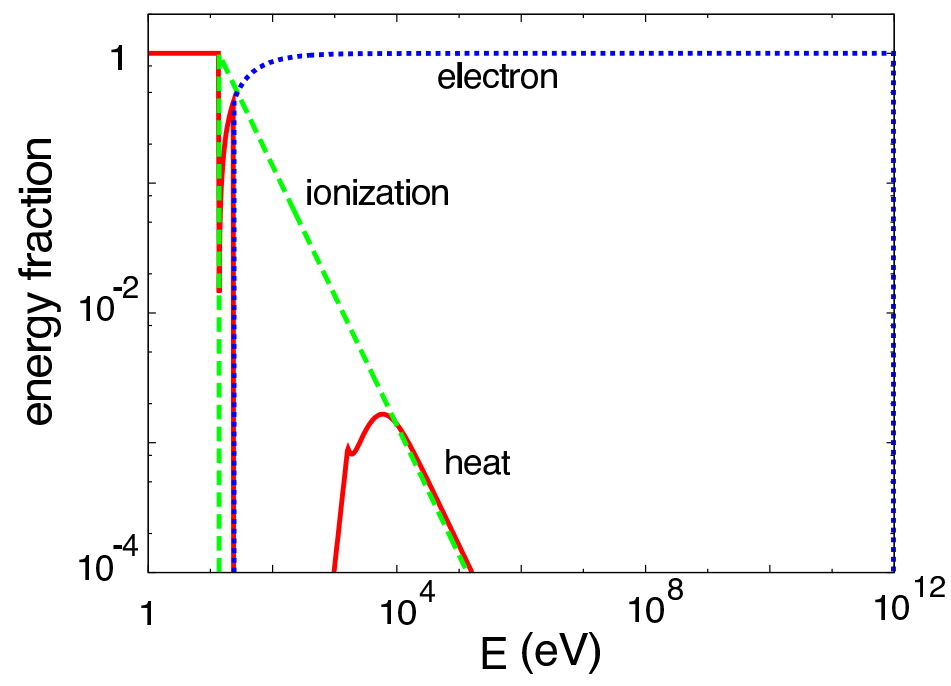

Figure 7. Photon energy degradation with $x_{e}=0$ and $1+z=1000$. Solid line represents $\chi_{h}$, dashed line represents $\chi_{i}$ and dotted line represents the fraction of electron energy which energy is larger than $0.75 \mathrm{Ry}$.

one $\left(E_{\gamma}=7 / 4 \mathrm{Ry}\right)$ corresponds to the sum of the threshold energy of photoionization and that of electron impact excitation. In that case the energy of the incident photon goes into ionization and the electron produced in the ionization, and is not used for heating. (Of course the electron further interacts with background plasma and heats it. This will be taken account in the next section.) The rise of $\chi_{h}$ around $E_{\gamma} \sim 10^{4} \mathrm{eV}$ is due to Compton scattering. Unlike the case of incident electron, the fraction of electron energy dominates even at low energy. This is because energetic electrons are produced through photoionization at low energy and Compton scattering at high energy.

The increase of ionization fraction causes the increases $\chi_{h}$ especially at low energy. This is because photoionization and Compton scattering determine the degradation 


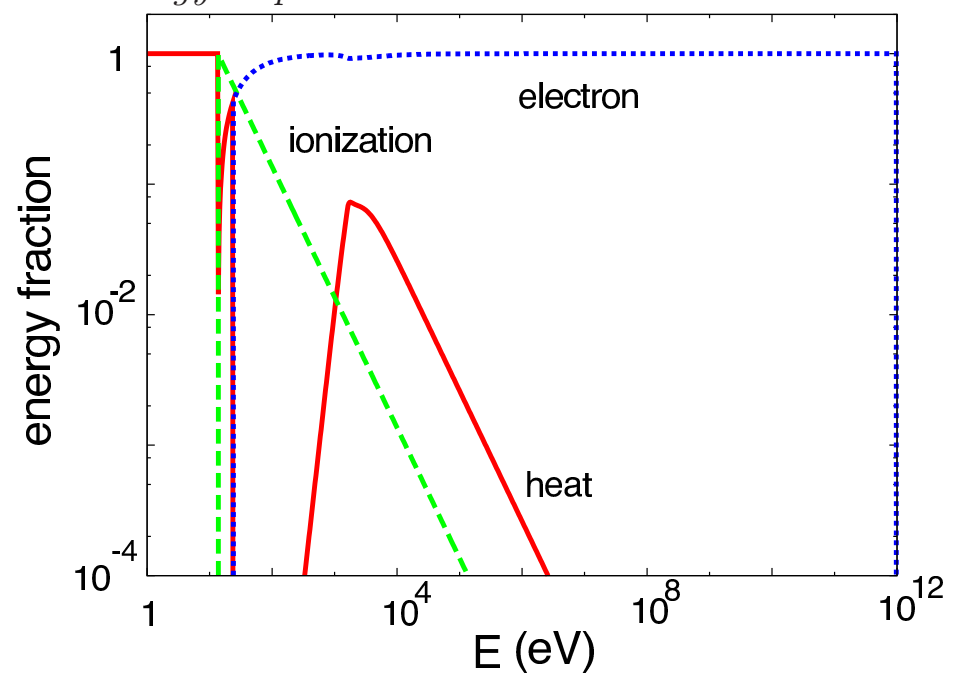

Figure 8. Photon energy degradation with $1-x_{e}=10^{-2}$ and $1+z=1000$. Solid line represents $\chi_{h}$, dashed line represents $\chi_{i}$ and dotted line represents the fraction of electron energy which energy is larger than $0.75 \mathrm{Ry}$.

of electron energy at low energy. The effect of photoionization decreases as ionization fraction increases, and hence $\chi_{h}$ increases. Since the photoionization rate is proportional to $\left(1-x_{e}\right)$, the effect of photoionization becomes small as $x_{e}$ approaches 1 , which leads to relative enhance of Compton scattering and increases $\chi_{h}$. Note that we use the the baryon density instead of the electron density when we calculate the energy losses due to Compton scattering. This is because Compton scattering only becomes important when $E_{\gamma}$ is sufficiently larger than Ry and the interaction is insensitive to whether an electron is bound or not [2]. As redshift increases, photon-photon scattering and double photon pair creation become important. These processes, however, become dominant at very high energy as seen in Fig. 6. This effect is not appeared until the energy deposition of electron and positron taken into account.

\subsection{Positron}

Positrons are produced by pair production in electric field of nuclei and double photon pair creation. Therefore, we should take the energy degradation of positrons into account. Although the energy degradation of positrons is almost the same as that of electron, there are two differences between electron and positron. One is the sign of its charge. The other is the indistinguishability between a primary electron and a secondary electron in the processes where target is an electron or an atomic hydrogen. These differences become less important at high energy. For simplicity, we assume positrons lose their energy just like electrons in this paper. Besides, positrons finally annihilate with background electrons through either free annihilation or the formation and decay of positronium [30]. It depends on the temperature, density and state of ionization of the background electron which process is dominant. Roughly speaking, most of the positrons undergo annihilation after the significant loss of its energy. Therefore, we assume that a 


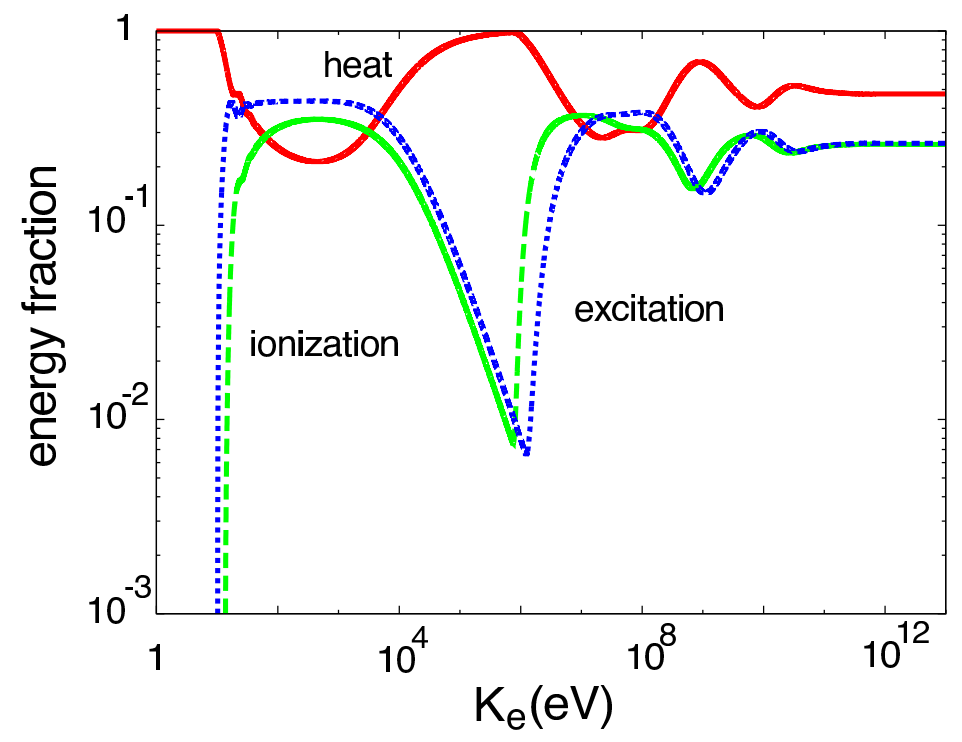

Figure 9. Electron energy degradation with $x_{e}=10^{-3}$ and $1+z=1000$. Solid line represents $\chi_{h}$, dashed line represents $\chi_{i}$ and dotted line represents $\chi_{e x}$.

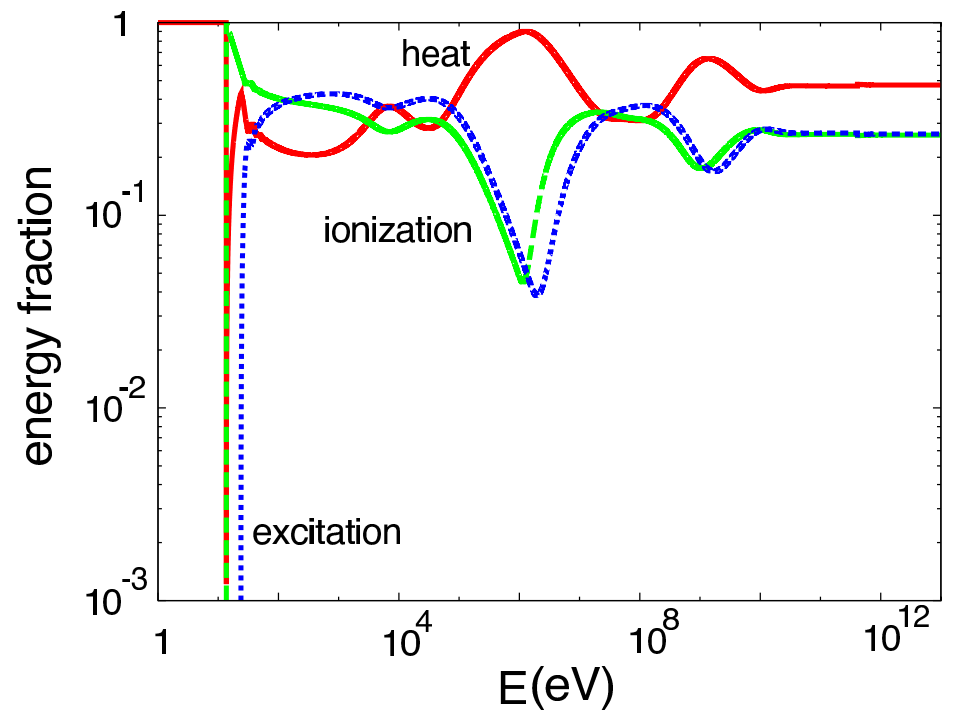

Figure 10. Photon energy degradation with $x_{e}=10^{-3}$ and $1+z=1000$. Solid line represents $\chi_{h}$, dashed line represents $\chi_{i}$ and dotted line represents $\chi_{e x}$.

positrons forms a positronium with a background electron after losing almost all of its energy and decay. The positronium annihilates to two photons (each $0.511 \mathrm{MeV}$ ) $25 \%$ of the time, and to three photons (each less than $0.511 \mathrm{MeV}$ ) $75 \%$ of the time. The energy spectrum from the three-photon annihilation is described in Appendix A. 


\section{Results}

As previously mentioned, we should calculate the evolution of the energy of charged particles and photons simultaneously. In Fig. 9 and Fig. 10, we show the fraction of heat, excitation and ionization when the primary particle is electron and photon respectively. Let us examine these figures.

First, we consider the electron case. When the energy of the electron is small $\left(K_{e}<10^{4} \mathrm{eV}\right)$ and the ionization frcraction is not large, the fractions of heat, excitation and ionization are roughly the same order. This is because relative smallness of ionization fraction makes the effect of Coulomb loss comparable with that of excitation and ionization of atomic hydrogen. The fraction of heat increases as ionization fraction increases and vice versa. For larger electron energy $\left(K_{e}>10^{4} \mathrm{eV}\right)$, the dominant energy loss mechanism is the inverse Compton scattering. If the electron is non-relativistic, the energy of scattered-up photons is so small that these photons only contribute to heating of the background particles. As a consequence, the fraction of heat reaches near unity. When an electron is relativistic, the energy of scattered-up photons exceeds the threshold energy of ionization of atomic hydrogen. In this case, it is necessary to estimate the contribution of these photons. It is seen that the fractions of excitation and ionization are a little larger than that of heat with low photon energy in Fig. 10. For this reason, all of these fractions becomes almost the same amount. The oscillating structure of the $\chi^{\prime}$ 's around $K_{e} \simeq 10^{8} \sim 10^{10} \mathrm{eV}$ reflects that of scattered-up photon around $E_{\gamma} \simeq 10^{5} \sim 10^{7} \mathrm{eV}$. When the energy of electron becomes ultra-relativistic, these oscillating structure is averaged out and vanishes.

Next, let us consider the photon case. When the energy of the incident photon is small $\left(E_{\gamma}<10^{3} \mathrm{eV}\right)$, the fractions of excitation and ionization are larger than that of heat. This is because the dominant energy loss mechanism is photoelectron effect unless ionization fraction is very close to unity. In this case, a photon ionizes an atomic hydrogen and emits a photoelectron whose energy is almost the same as the primary photon. Therefore, the behavior of photons is very similar to that of electrons at low energy. As photon energy increases, Compton scattering becomes the dominant energy loss. When the photon energy is larger than the electron mass, the forward scattering becomes dominant and the energy of the recoil electron approaches to that of the photon. In other words, Compton scattering produces a recoil electron with energy same as the incident photon. Therefore, the behavior of photons traces that of electrons in this energy region.

When photon energy is larger than $10^{8} \mathrm{eV}$, the effect of pair-production in matter, photon-photon scattering and double photon pair creation can not be neglected. However, it is hard to explain the influence of these processes because the most dominant process among the three depends on redshift and photon energy, and the shape of the spectrum of secondary particles is very sensitive to primary photon energy. At high energy, however, the fraction of heat, excitation and ionization become constant values just like electron. As high energy photons are closely related with electrons, these 


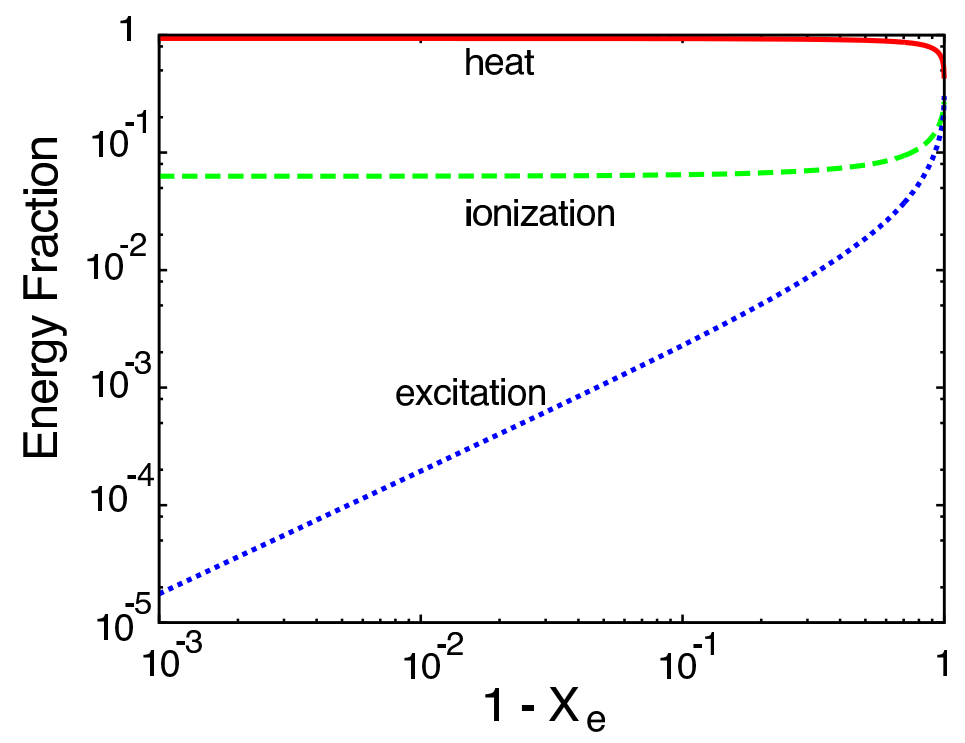

Figure 11. Electron and photon energy degradation with $1+z=1000$. Solid line represents $\chi_{h}$, dashed line represents $\chi_{i}$ and dotted line represents $\chi_{e x}$.

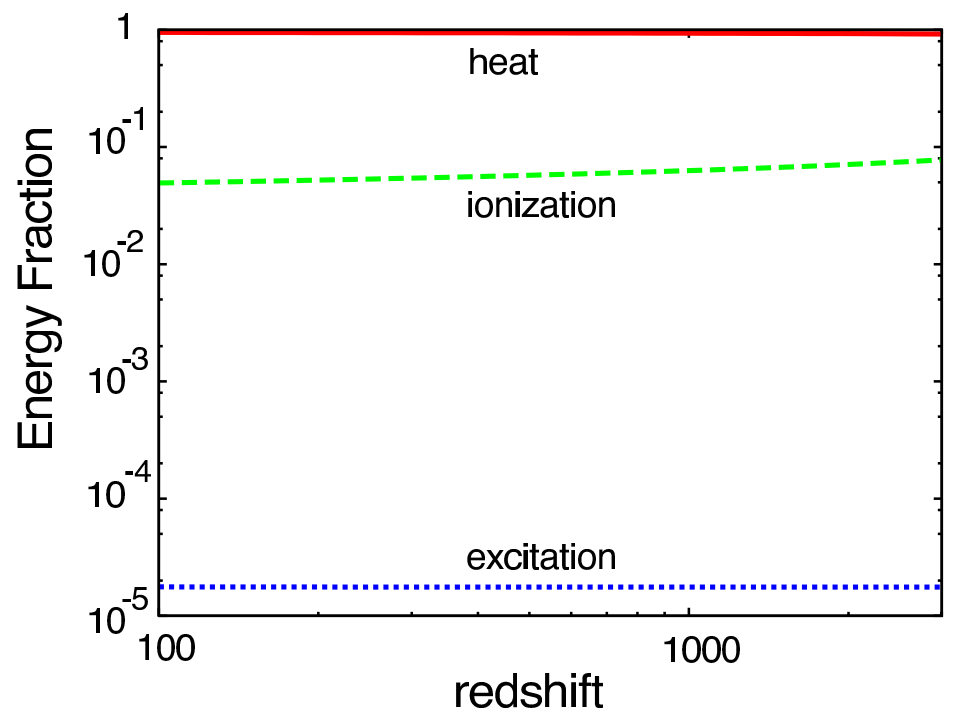

Figure 12. Electron and photon energy degradation with $x_{e}=10^{-3}$. Solid line represents $\chi_{h}$, dashed line represents $\chi_{i}$ and dotted line represents $\chi_{e x}$.

constants will become the almost same value in either case.

To see how ionization fraction $x_{e}$ and redshift $z$ have influence on the energy degradation, we plot the fractions of heat, ionization and excitation as function of $1-x_{e}$ and $1+z$ in Figs. 11 and 12, repectively. Here we have taken $10^{12} \mathrm{eV}$ for the initial energy of electrons or photons. This is because all the fraction become constant values at this energy. Unless ionization fraction is very small, $\chi_{h}$ and $\chi_{i}$ are almost independent of it. This is because $\chi_{i}$ is mainly determined by photoionization due to low energy secondary photons in this case and the collision frequency of photoionization is much 
larger than that of Compton scattering at low energy[Fig. 6]. Therefore the effect of the change of ionization fraction is almost irrelevant. However, the fraction of excitation $\chi_{e}$ is very sensitive to ionization fraction since the collision frequency of electron impact excitation is in proportion to $1-x_{e}$ while the competing processes (inverse Compton and Coulomb loss) are independent of $1-x_{e}$. The fraction of heat $\chi_{h}$ does not depend on $1-x_{e}$ since $\chi_{i}$ is independent of $1-x_{e}$ and $\chi_{e x}$ is very small quantity. (Please notice that $\chi_{i}+\chi_{e x}+\chi_{h}=1$.) When the ionization fraction is very small, the effect of Coulomb scattering is weakened and these fractions become the same order. Unlike ionization fraction, the change of redshift seems to have no influence on the energy degradation. This is because the change of redshift effects which process is dominant at high energy, but is almost irrelevant at low energy. $\chi_{i}$ and $\chi_{e}$ are determined by secondary low energy particles and these fractions are nearly independent of redshift.

\section{Conclusions}

We have carried out detailed calculations of the fractions of the initial energy of the injected electron or photon which are used to heat, ionize and excite background plasma in the early universe. In the high energy limit $\left(E>10^{12} \mathrm{GeV}\right)$, we have shown that the fractions are alomost independent of the initial energy. Our calculations are valid up to $\mathrm{TeV}$ and can be applied to various cosmological and astrophysical situations such as dark matter decay/annihilation, which will be studied in a separete paper.

Acknowledgement: This work was supported in part by the Grant-in-Aid for Scientific Research from the Ministry of Education, Science, Sports, and Culture of Japan, No 14102004 (M.K.). This work was supported by World Premier International Research

Center InitiativeiWPI Initiative), MEXT, Japan. This work was also supported in part by JSPS-AF Japan-Finland Bilateral Core Program.

\section{Appendix A. Relevant Cross Sections}

In this appendix, we show the cross sections and energy loss rates adopted in this paper.

\section{Appendix A.1. Electron}

Appendix A.1.1. Excitation of $H$ We have adopted the almost same cross sections for atomic hydrogen and Coulomb losses as [1. For the cross sections for the electron impact excitation of atomic hydrogen, we have adopted [7]. At high energy, we have used the Bethe approximation reviewed in [8, 9, 12, 23].

$$
\begin{aligned}
\sigma_{2 p}(\mathrm{NR}) & =\frac{4 \pi a_{0}^{2}}{T / \mathrm{Ry}}\left[M_{2 p}^{2} \ln \left(\frac{4 C_{2 p} T}{\mathrm{Ry}}\right)+\frac{\gamma_{2 p}}{T / \mathrm{Ry}}\right], \\
\sigma_{2 p}(\mathrm{R}) & =\frac{8 \pi a_{0}^{2}}{m v^{2} / \mathrm{Ry}} M_{2 p}^{2}\left[\ln \left(\frac{\beta^{2}}{1-\beta^{2}}\right)-\beta^{2}+\ln C_{2 p}+11.2268\right],
\end{aligned}
$$


where $a_{0}$ is the Bohr radius, Ry is the Rydberg energy and $T=m v^{2} / 2$ represents the kinetic energy. The numerical coefficients are $\ln C_{2 p}=-0.89704, \gamma_{2 p}=0.207985$ and $M_{2 p}^{2}=0.55493$. "NR" and "R" mean non-relativistic and relativistic respectively.

$$
\begin{aligned}
\sigma_{2 s}(\mathrm{NR}) & =\frac{4 \pi a_{0}^{2}}{T / \mathrm{Ry}}\left[b_{2 s}+\frac{\gamma_{2 s}}{T / \mathrm{Ry}}\right], \\
\sigma_{2 s}(\mathrm{R}) & =\frac{8 \pi a_{0}^{2}}{m v^{2} / \mathrm{Ry}} b_{2 s},
\end{aligned}
$$

where $b_{2 s}=0.11986$ and $\gamma_{2 s}=-0.3125$.

$$
\begin{aligned}
\sigma_{n=3}(\mathrm{NR}) & =\frac{4 \pi a_{0}^{2}}{T / \mathrm{Ry}}\left[M_{3}^{2} \ln \left(\frac{4 C_{3} T}{\mathrm{Ry}}\right)\right], \\
\sigma_{n=3}(\mathrm{R}) & =\frac{8 \pi a_{0}^{2}}{m v^{2} / \mathrm{Ry}} M_{3}^{2}\left[\ln \left(\frac{\beta^{2}}{1-\beta^{2}}\right)-\beta^{2}+\ln C_{3}+11.2268\right],
\end{aligned}
$$

where $M_{3}^{2}=8.8989 \times 10^{-2}$ and $\ln C_{3}=-0.2724$.

For the excitation to $n>3$, we subtract Eq. (A.1) Eq. (A.6) from total excitation cross section.

$$
\begin{aligned}
\sigma_{e x}(\mathrm{NR}) & =\frac{4 \pi a_{0}^{2}}{T / \mathrm{Ry}}\left[M_{e x}^{2} \ln \left(\frac{4 C_{e x} T}{\mathrm{Ry}}\right)+\frac{\gamma_{e x}}{T / \mathrm{Ry}}\right], \\
\sigma_{e x}(\mathrm{R}) & =\frac{8 \pi a_{0}^{2}}{m v^{2} / \mathrm{Ry}} M_{e x}^{2}\left[\ln \left(\frac{\beta^{2}}{1-\beta^{2}}\right)-\beta^{2}+\ln C_{e x}+11.2268\right],
\end{aligned}
$$

where $\ln C_{e x}=-0.5780, \gamma_{e x}=-0.120575$ and $M_{e x}^{2}=0.7166$.

Appendix A.1.2. Ionization of $H$ For the ionization cross section, we have adopted the following differential cross section.

$$
\frac{d \sigma_{i}(E, \epsilon)}{d \epsilon}=\frac{A(E)}{1+(\epsilon / \bar{\epsilon})^{2}} \quad \text { for } \quad 0 \leq \epsilon \leq \frac{1}{2}(E-I)
$$

where $E$ is the incident electron energy and $\epsilon$ is the energy of the ejected electron. We choose $\bar{\epsilon}=8 \mathrm{eV}$. In Eq. (A.9), a value of 2 is different from that of 2.1 originally suggested by [10]. This is why Eq. (A.9) can be analytically integrated. Two parameters $A(E)$ and $\bar{\epsilon}$ are related to the total ionization cross section $\sigma_{i}(E)$

$$
\begin{aligned}
& A(E)=\frac{\sigma_{i}(E)}{\bar{\epsilon}}\left[\tan ^{-1} X(E)\right]^{-1}, \\
& X(E)=\frac{E-I}{2 \bar{\epsilon}},
\end{aligned}
$$

where $I$ is the ionization potential. The total ionization cross section for atomic hydrogen had been measured by [11] in the range 14.6-4000 eV. Above $4000 \mathrm{eV}$, we used Bethe approximation [8, 12.

$$
\begin{aligned}
\sigma_{i}(\mathrm{NR}) & =\frac{4 \pi a_{0}^{2}}{T / \mathrm{Ry}}\left[M_{i}^{2} \ln \left(\frac{4 C_{i} T}{\mathrm{Ry}}\right)+\frac{\gamma_{i}}{T / \mathrm{Ry}}\right] \\
\sigma_{i}(\mathrm{R}) & =\frac{8 \pi a_{0}^{2}}{m v^{2} / \mathrm{Ry}} M_{i}^{2}\left[\ln \left(\frac{\beta^{2}}{1-\beta^{2}}\right)-\beta^{2}+\ln C_{i}+11.2268\right],
\end{aligned}
$$


where $M_{i}^{2}=0.2834, \ln C_{i}=3.048$ and $\gamma_{i}=-1.6294+\ln (\mathrm{Ry} / T)$.

Appendix A.1.3. $e^{-}-H$ Collision For electron-hydrogen momentum transfer cross sections at low energies, we have adopted the results of [13, 14]. The momentum loss cross section is described by

$$
\sigma_{m t}=\frac{\pi a_{0}^{2}}{T / \mathrm{Ry}} \sum_{l=0}\left[3 \sin ^{2}\left(\eta_{l+1}^{-}-\eta_{l}^{-}\right)+\sin ^{2}\left(\eta_{l+1}^{+}-\eta_{l}^{+}\right)\right]
$$

where $\eta_{l}^{+}$and $\eta_{l}^{-}$are the phase shift computed in [15, 16]. The cross section at 100, 200 and $300 \mathrm{eV}$ were calculated in [17]. Cross sections at other energies were derived by interpolation and extrapolation. The energy loss due to electron-hydrogen momentum transfer is described by

$$
\left[\frac{-d E}{d t}\right]_{m t}=\frac{2 m_{e} E}{m_{p}} n_{H} v_{e} \sigma_{m t}(E)
$$

where $m_{p}$ is the proton mass.

Appendix A.1.4. Coulomb Collision Incident electrons lose their energies due to elastic collisions with background electrons and photons. Energy loss is dominated by electrons at low energy since Coulomb cross sections are much larger than Compton cross sections. However, energy loss is dominated by photons at high energy since the number density of photons is much larger than that of electrons.

For the energy loss due to Coulomb collisions with background electrons, we have adopted the following analytical formula [18].

$$
\left[\frac{-d E}{d t}\right]_{C l}=\frac{2.0 \times 10^{-4} n_{e}^{0.97}}{E^{0.44}}\left(\frac{E-E_{e}}{E-0.53 E_{e}}\right)^{2.36} \mathrm{eV} \cdot \mathrm{s}^{-1}
$$

where $E$ is the incident electron energy in $\mathrm{eV}, E_{e}$ is the background electron energy in $\mathrm{eV}$ and $n_{e}$ is the electron number density in $\mathrm{cm}^{-3}$.

Appendix A.1.5. Inverse Compton Scattering An important quantity which characterizes the behavior of inverse Compton scattering is $\gamma E_{C M B}$ (photon energy in the electron's rest frame) where $E_{C M B}$ is the energy of CMB photon. If $\gamma E_{C M B}$ is much less than $m_{e}$, Thomson scattering approximation is valid. Otherwise, Klein-Nishina cross section should be used.

For inverse Compton scatterin with $\gamma E_{C M B} \ll m_{e}$, the energy spectrum of scattered photon is obtained by [4] in the limit $\beta \rightarrow 1$. However, we should keep $\beta$ so as not to spoil the validity of Thomson approximation. After some tedious calculations, the number of collisions per unit time and unit scattered photon energy $\left(E_{\gamma}\right)$ is given by

$$
\frac{d^{2} N}{d t d x}=\sigma_{T} c n\left(E_{C M B}\right) d E_{C M B} f(x),
$$


where $\sigma_{T}$ is the Thomson cross section and $n\left(E_{C M B}\right)$ is the differential number density of CMB photons and $x=E_{\gamma} / E_{C M B}$. The expressions for $f(x)$ is given by

$$
\begin{aligned}
f(x) & =\frac{3}{16 \gamma^{4} \beta^{4}}\left[-\left(1+\beta^{2}\right)\left(\frac{1}{1+\beta}-\frac{x}{1-\beta}\right)+\left(x^{2}(1+\beta)-x(1-\beta)\right)\right. \\
& \left.-2 x \ln \left(x \frac{1+\beta}{1-\beta}\right)\right] \quad \text { for } \quad \frac{1-\beta}{1+\beta} \leq x \leq 1, \\
f(x) & =\frac{3}{16 \gamma^{4} \beta^{4}}\left[-\left(1+\beta^{2}\right)\left(\frac{x}{1+\beta}-\frac{1}{1-\beta}\right)+\left(x(1+\beta)-x^{2}(1-\beta)\right)\right. \\
& \left.-2 x \ln \left(\frac{1}{x} \frac{1+\beta}{1-\beta}\right)\right] \quad \text { for } \quad 1 \leq x \leq \frac{1+\beta}{1-\beta} .
\end{aligned}
$$

The coefficient is determined so that $\int f(x) d x$ is equal to unity. In the limit $\beta \rightarrow 1$, Eq. (A.19) corresponds to the result of [4]. The number of collisions per unit time and the energy loss rate can be easily obtained.

$$
\begin{aligned}
& \int \frac{d^{2} N}{d t d x} d x=\sigma_{T} c n\left(E_{C M B}\right) d E_{C M B}=\sigma_{T} c n_{C M B} \\
& \int \frac{d^{2} N}{d t d x}\left(E_{\gamma}-E_{C M B}\right) d x=\frac{4}{3} \sigma_{T} c n\left(E_{C M B}\right) E_{C M B} d E_{C M B} \gamma^{2} \beta^{2} \\
&=\frac{4}{3} \sigma_{T} c U_{C M B} \gamma^{2} \beta^{2},
\end{aligned}
$$

where $n_{C M B}$ and $U_{C M B}$ are the number and energy density of CMB photons.

For inverse Compton scattering with $\gamma E_{C M B} \geq m_{e}$, we should use Klein-Nishina cross section instead of Thomson cross section. The number of collisions per unit time and unit scattered photon energy is given by [22]

$$
\begin{aligned}
\frac{d^{2} N}{d t d \alpha^{\prime}} & =\frac{2 \pi r_{e}^{2} c}{\alpha \gamma^{2}}\left[2 q \ln q+(1+2 q)(1-q)+\frac{1}{2} \frac{(4 \alpha \gamma q)^{2}}{1+4 \alpha \gamma q}(1-q)\right] \\
& \times n\left(E_{C M B}\right) d E_{C M B} \quad \text { for } \quad \alpha \leq \alpha^{\prime} \leq \frac{4 \alpha \gamma^{2}}{1+4 \alpha \gamma}
\end{aligned}
$$

where $\alpha=E_{C M B} / m_{e}, \alpha^{\prime}=E_{\gamma} / m_{e}$ and $q=\alpha^{\prime} / 4 \alpha \gamma^{2}\left(1-\alpha^{\prime} / \gamma\right)$. The number of collisions per unit time can be obtained by integrating Eq. (A.22). We shall assume that Eq. (A.22) is valid for $0<q<1$, even though Eq. (A.22) is quite invalid for $0<q<1 / 4 \gamma^{2}$. The contribution from the region $0<q<1 / 4 \gamma^{2}$ is $O\left(1 / \gamma^{2}\right)$ and is negligible since $E_{C M B}$ is much less than $m_{e}$ [22]. The number of collisions per unit time is given by 32

$$
\int \frac{d^{2} N}{d t d \alpha^{\prime}} d \alpha^{\prime} \simeq \int_{0}^{1} \frac{d^{2} N}{d t d q} d q=\sigma_{T} c \psi_{1}(s) n\left(E_{C M B}\right) d E_{C M B},
$$

where

$$
\psi_{1}(s)=\frac{3}{2 s^{2}}\left[\left(s+9+\frac{8}{s}\right) \ln (1+s)-8-\frac{2 s+s^{2}}{2+2 s}+4 \operatorname{Li}_{2}(-s)\right],
$$

and $s=4 \alpha \gamma$. The function $\mathrm{Li}_{2}(x)$ is the dilogarithm

$$
\operatorname{Li}_{2}(x) \equiv-\int_{0}^{x} d z \frac{\ln (1-z)}{z} \text {. }
$$


The energy loss rate is given by [22]

where

$$
\int E_{\gamma} \frac{d^{2} N}{d t d \alpha^{\prime}} d \alpha^{\prime} \simeq \frac{4}{3} \sigma_{T} c \gamma^{2} \psi_{2}(s) n\left(E_{C M B}\right) E_{C M B} d E_{C M B}
$$

$$
\begin{aligned}
\psi_{2}(s) & =\frac{9}{s^{3}}\left[\left(\frac{s}{2}+6+\frac{6}{s}\right) \ln (1+s)\right. \\
& \left.-\frac{6+13 s+8 s^{2}+11 s^{3} / 12}{(1+s)^{2}}+2 \operatorname{Li}_{2}(-s)\right] .
\end{aligned}
$$

\section{Appendix A.2. Photon}

Appendix A.2.1. Photoionization The absorption of X-rays and $\gamma$-rays is studied in detail in 21]. Incident photons are mainly absorbed by hydrogen atoms and eject photoelectrons at low energies. The photoionization cross section for atomic hydrogen was reviewed in [19, 20].

$$
\begin{aligned}
\sigma_{K}(\mathrm{NR}) & =\frac{64 \pi \sigma_{T}}{\alpha^{3}}\left(\frac{I}{h \nu}\right)^{4} \frac{\exp \left(-4 \eta \cot ^{-1} \eta\right)}{1-\exp (-2 \pi \eta)} \\
\sigma_{K}(\mathrm{R}) & =\frac{3 \sigma_{T} \alpha^{4}}{4}\left(\frac{m_{e}}{h \nu}\right)^{5}\left[\gamma^{2}-1\right]^{3 / 2} \\
& \times\left[\frac{4}{3}+\frac{\gamma(\gamma-2)}{\gamma+1}\left(1-\frac{1}{2 \gamma \sqrt{\gamma^{2}-1}} \ln \left(\frac{\gamma+\sqrt{\gamma^{2}-1}}{\gamma-\sqrt{\gamma^{2}-1}}\right)\right)\right]
\end{aligned}
$$

where $h \nu$ is the incident photon energy, $\alpha$ is the fine-structure constant, $I$ is ionization energy, $\eta=1 / \sqrt{h \nu / I-1}$ and $\gamma=\left(h \nu+m_{e}\right) / m_{e}$. The cross sections above are just halves of [19, 20]. This is because there is only one electron in $\mathrm{K}$ shell in the case of hydrogen.

Appendix A.2.2. Compton Scattering Incident photons interact with background electrons through Compton scattering. If photon energy is sufficient small, the energy of recoil electron is below the threshold energy of excitation and ionization of atomic hydrogen. Therefore the energy transferred to the recoil electron can be regarded as heating. Besides, a photon loses only a small fraction of its energy per scattering. The energy loss due to Compton scattering is described by [21]

$$
\left[\frac{-d E}{d t}\right]_{\text {Compton }}=m_{e} n_{e} c \sigma_{T} x^{2} g(x),
$$

where $x=h \nu / m_{e}$ and

$$
\begin{aligned}
g(x) & =\frac{3}{8}\left[\frac{(x-3)(x+1)}{x^{4}} \ln (1+2 x)\right. \\
& \left.+\frac{2\left(3+17 x+31 x^{2}+17 x^{3}-\frac{10}{3} x^{4}\right)}{x^{3}(1+2 x)^{3}}\right], \\
& \simeq 1, \quad \text { for } \quad x \ll 1 ; \quad \text { for } \quad x \gg 1 ; \\
& \simeq \frac{3}{8 x^{2}}\left(\ln 2 x-\frac{5}{6}\right), \quad \text { in }
\end{aligned}
$$


If photon energy is as large as the electron mass, a photon loses a sizable fraction of its energy per scattering. In this case, it is necessary to calculate the energy distribution of the recoil electrons. The cross section is given by the following Klein-Nishina formula:

$$
\begin{gathered}
\frac{d \sigma}{d \epsilon}(h \nu)=\frac{3 \sigma_{T}}{8} \frac{m_{e}}{(h \nu)^{2}}\left[\frac{h \nu}{\epsilon}+\frac{\epsilon}{h \nu}+\left(\frac{m_{e}}{\epsilon}-\frac{m_{e}}{h \nu}\right)^{2}-2\left(\frac{m_{e}}{\epsilon}-\frac{m_{e}}{h \nu}\right)\right] \\
\text { for } \quad \frac{m_{e}}{m_{e}+2 h \nu} h \nu \leq \epsilon \leq h \nu,
\end{gathered}
$$

where $\epsilon$ is the scattered photon energy.

Appendix A.2.3. Pair Creation If photon energy is larger than $2 m_{e}$, it is possible to create electron-positron pair. The energy and momentum conservation, however, are only possible if another particle is present. The differential cross section for pair creation in nuclei is given by the Bethe-Heitler formula [19, 24] :

$$
\begin{aligned}
\frac{d \sigma}{d E_{+}} & =\alpha r_{e}^{2} \frac{p_{+} p_{-}}{E_{\gamma}^{2}}\left\{-\frac{4}{3}-2 E_{+} E_{-} \frac{p_{+}^{2}+p_{-}^{2}}{p_{+}^{2} p_{-}^{2}}+m_{e}^{2}\left(\frac{E_{+} l_{-}}{p_{-}^{3}}+\frac{E_{-} l_{+}}{p_{+}^{3}}-\frac{l_{+} l_{-}}{p_{+} p_{-}}\right)\right. \\
& +L\left[\frac{E_{\gamma}^{2}}{p_{+}^{3} p_{-}^{3}}\left(E_{+}^{2} E_{-}^{2}+p_{+}^{2} p_{-}^{2}\right)-\frac{8}{3} \frac{E_{+} E_{-}}{p_{+} p_{-}}-\frac{m_{e}^{2} E_{\gamma}}{2 p_{+} p_{-}}\right. \\
& \left.\left.\times\left(\frac{E_{+} E_{-}-p_{-}^{2}}{p_{-}^{3}} l_{-}+\frac{E_{+} E_{-}-p_{+}^{2}}{p_{+}^{3}} l_{+}+\frac{2 E_{\gamma} E_{+} E_{-}}{p_{+}^{2} p_{-}^{2}}\right)\right\}\right],
\end{aligned}
$$

where

$$
\begin{aligned}
& p_{ \pm}=\sqrt{E_{ \pm}^{2}-m_{e}^{2}}, \\
& L=\ln \frac{E_{+} E_{-}+p_{+} p_{-}+m_{e}^{2}}{E_{+} E_{-}-p_{+} p_{-}+m_{e}^{2}}, \\
& l_{ \pm}=\ln \frac{E_{ \pm}+p_{ \pm}}{E_{ \pm}-p_{ \pm}},
\end{aligned}
$$

and $E_{ \pm}$is the energy of positron (electron) energy. The analytical expression for cross section is given by [24, 25]

$$
\begin{aligned}
\sigma & =\alpha r_{e}^{2}\left\{\frac{28}{9} \ln 2 k-\frac{218}{27}+\left(\frac{2}{k}\right)^{2}\left[6 \ln 2 k-\frac{7}{2}+\frac{2}{3} \ln ^{3} 2 k-\ln ^{2} 2 k\right.\right. \\
& \left.-\frac{\pi^{2}}{3} \ln 2 k+\frac{\pi^{2}}{6}+2 \zeta(3)\right]-\left(\frac{2}{k}\right)^{4}\left[\frac{3}{16} \ln 2 k+\frac{1}{8}\right] \\
& \left.-\left(\frac{2}{k}\right)^{6}\left[\frac{29}{9 \times 256} \ln 2 k-\frac{77}{27 \times 512}\right]+\cdots\right\} \text { for } k>4
\end{aligned}
$$

where $k=E_{\gamma} / m_{e}$. Convenient approximate formulas are given by [26] which are valid for $k \leq 20$,

$$
\frac{d \sigma}{d x}=\alpha r_{e}^{2} \phi_{0} z\left[1+0.135\left(\phi_{0}-0.52\right) z\left(1-z^{2}\right)\right]
$$

where

$$
x=\frac{E_{+}-m_{e}}{E_{\gamma}-2 m_{e}}
$$


Electron and Photon Energy Deposition in Universe

$$
z=2 \sqrt{x(1-x)}
$$

and $\phi_{0}$ is the differential cross section for equal partition of energy, $E_{+}=E_{-}=E_{\gamma} / 2$. The second term in the square bracket should be dropped when it becomes negative (below $k=4.2$ ). $\phi_{0}$ is given by

$$
\phi_{0}=\left(1-\gamma_{1}\right)\left[\frac{1}{3}\left(4-\gamma_{1}^{2}\right)\left(L_{1}-1\right)-\gamma_{1}^{2} \alpha_{1}\left(\alpha_{1}-1\right)-\gamma_{1}^{4} \alpha_{1}\left(L_{1}-\alpha_{1}\right)\right]
$$

where

$$
\begin{aligned}
\gamma_{1} & =\frac{2}{k}, \\
L_{1} & =\frac{2}{1-\gamma_{1}^{2}} \ln \left(\frac{k}{2}\right), \\
\alpha_{1} & =\frac{1}{\sqrt{1-\gamma_{1}^{2}}} \ln \left[\frac{k}{2}+\sqrt{\left(\frac{k}{2}\right)^{2}-1}\right] .
\end{aligned}
$$

We get from Eq. (A.40) for the total cross section

$$
\begin{aligned}
\sigma & =\frac{\pi}{4} \alpha r_{e}^{2} \phi_{0} & \text { for } & k<4.2, \\
& =\alpha r_{e}^{2}\left(0.776 \phi_{0}+0.018 \phi_{0}^{2}\right) & \text { for } & k>4.2 .
\end{aligned}
$$

Appendix A.2.4. Photon-Photon Scattering If the photon energy is below the effective threshold energy of the double photon pair creation, photon-photon scattering $(\gamma \gamma \rightarrow$ $\gamma \gamma$ ) process becomes significant. The photon-photon scattering rate for $E_{\gamma} E_{C M B} \leq m_{e}^{2}$ is given by [27]

$$
P\left(E_{\gamma}\right)=3.33 \times 10^{11}\left(\frac{T_{C M B}}{m_{e}}\right)^{6}\left(\frac{E_{\gamma}}{m_{e}}\right)^{3} \mathrm{~s}^{-1} .
$$

Normalized distribution of secondary photons of energy $E_{\gamma}^{\prime}, p\left(E_{\gamma}^{\prime}, E_{\gamma}\right)$, is given by

$$
p\left(E_{\gamma}^{\prime}, E_{\gamma}\right)=\frac{20}{7} \frac{1}{E_{\gamma}}\left[1-\frac{E_{\gamma}^{\prime}}{E_{\gamma}}+\left(\frac{E_{\gamma}^{\prime}}{E_{\gamma}}\right)^{2}\right]^{2} \quad \text { for } \quad 0 \leq E_{\gamma}^{\prime} \leq E_{\gamma} .
$$

The distribution $p\left(E_{\gamma}^{\prime}, E_{\gamma}\right)$ satisfies

$$
\begin{aligned}
& \frac{1}{2} \int_{0}^{E_{\gamma}} p\left(E_{\gamma}^{\prime}, E_{\gamma}\right) d E_{\gamma}^{\prime}=1, \\
& \int_{0}^{E_{\gamma}} p\left(E_{\gamma}^{\prime}, E_{\gamma}\right) E_{\gamma}^{\prime} d E_{\gamma}^{\prime}=E_{\gamma} .
\end{aligned}
$$

The above formulas are not valid for a larger value of $E_{\gamma}$. However, photon-photon scattering is not significant for high energy photons since double photon pair creation is the dominant process. Therefore, instead of using exact formulas, we simply neglect photon-photon scattering for $E_{\gamma} E_{C M B}>m_{e}^{2}$. 
Appendix A.2.5. Double Photon Pair Creation For high energy photon, double photon pair creation $\left(\gamma \gamma \rightarrow e^{+} e^{-}\right)$is the dominant process. The total cross section for double photon pair creation is given by [28]

$$
\sigma=\frac{1}{2} \pi r_{e}^{2}\left(1-\beta^{2}\right)\left[\left(3-\beta^{4}\right) \ln \frac{1+\beta}{1-\beta}-2 \beta\left(2-\beta^{2}\right)\right],
$$

where $\beta$ is the electron (positron) velocity in the center-of-mass system. The relationship between $\beta$ and $E_{\gamma}, E_{C M B}$ and $\theta$ which is the angle between the momenta of the colliding photons is easily obtained:

$$
\begin{aligned}
& \beta=\sqrt{1-\frac{1}{s}}, \\
& s=\frac{E_{\gamma} E_{C M B}}{2 m_{e}^{2}}(1-\cos \theta) .
\end{aligned}
$$

Clearly, the threshold energy for double photon pair production is $E_{\gamma}=m_{e}^{2} / E_{C M B}$, head-on photon collision $(\theta=\pi, s=1)$. For calculation of the absorption probability, we should average the above cross section over the distributions for isotropically distributed photons 28, 32,

$$
\begin{aligned}
\sigma_{\text {ave }} & =\frac{1}{2} \int_{-1}^{1-2 m_{e}^{2} / E_{\gamma} E_{C M B}}(1-\cos \theta) \sigma d \cos \theta \\
& =\frac{3}{8} \sigma_{T}\left(\frac{m_{e}^{2}}{E_{\gamma} E_{C M B}}\right)^{2}\left[\frac{1+2 v+2 v^{2}}{1+v} \ln \omega-2 \sqrt{\frac{v}{1+v}}(1+2 v)\right. \\
& \left.+2 \ln ^{2}(1+\omega)-\ln ^{2} \omega+4 \operatorname{Li}_{2}\left(\frac{1}{1+\omega}\right)-\frac{\pi^{2}}{3}\right]
\end{aligned}
$$

where

$$
\begin{aligned}
& v=\frac{E_{\gamma} E_{C M B}}{m_{e}^{2}}-1>0, \\
& \omega=\frac{\sqrt{1+v}+\sqrt{v}}{\sqrt{1+v}-\sqrt{v}} .
\end{aligned}
$$

Differential spectra of electrons and positrons are given by [29]

$$
\begin{aligned}
\frac{d \sigma}{d E_{e}} & =\frac{\pi r_{e}^{2} m_{e}^{4}}{4 E_{\gamma}^{3} E_{C M B}^{2}}\left[\frac{4 E^{2}}{E_{e} E_{p}} \ln \frac{4 E_{C M B} E_{e} E_{p}}{m_{e}^{2} E}-\frac{8 E_{C M B} E}{m_{e}^{2}}\right. \\
& \left.+\frac{2\left(2 E_{C M B} E-m_{e}^{2}\right) E^{2}}{m_{e}^{2} E_{e} E_{p}}-\left(1-\frac{m_{e}^{2}}{E_{C M B} E}\right) \frac{E^{4}}{E_{e}^{2} E_{p}^{2}}\right],
\end{aligned}
$$

where $E_{e}\left(E_{p}\right)$ is the energy of electron (positron) and $E$ is the total energy, $E=$ $E_{e}+E_{p}=E_{\gamma}+E_{C M B}$. The limits of the variation of $E_{e}\left(E_{p}\right)$ is given by

$$
\frac{E}{2}\left(1-\sqrt{1-\frac{m_{e}^{2}}{E_{C M B} E}}\right) \leq E_{e} \leq \frac{E}{2}\left(1+\sqrt{1-\frac{m_{e}^{2}}{E_{C M B} E}}\right) .
$$




\section{Appendix A.3. Positronium}

Here, we show the energy spectrum from three-photon annihilation of positronium. The energy spectrum is continuous, as allowed by conservation of momentum. It has been calculated in [31] with photon energy $\eta$ normalized by electron mass,

$$
\begin{aligned}
F(\eta) & =\frac{2}{\pi^{2}-9}\left[\frac{\eta(1-\eta)}{(2-\eta)^{2}}-\frac{2(1-\eta)^{2}}{(2-\eta)^{3}} \ln (1-\eta)+\frac{2-\eta}{\eta}\right. \\
& \left.+\frac{2(1-\eta)}{\eta^{2}} \ln (1-\eta)\right] . \quad \text { for } \quad 0 \leq \eta \leq 1
\end{aligned}
$$

The function $F(\eta)$ is normalized so that

$$
\int_{0}^{1} d \eta F(\eta)=1 \text {. }
$$

[1] A. Dalgarno, M. Yan and W. Liu, Astrophys. J. Suppl. 125, 237 (1999).

[2] X. Chen and M. Kamionkowski, Phys. Rev. D70, 043502 (2004).

[3] D. N. Spergel et. al., Astrophys. J. Suppl. 170, 377 (2007).

[4] G. R. Blumenthal and R. J. Gould, Rev. Mod. Phys. 42, 237 (1970).

[5] A. Dalgarno and G. Lejeune, Planet. Space Sci., 19, 1653 (1971).

[6] V. L. Ginzburg and S. I. Syrovatskii, The Origin of Cosmic Rays, Pergamon Press, New York (1964).

[7] I. Shimamura, Sci. Papers Instit. Phys. Chem. Res. 82, 1 (1989).

[8] M. Inokuti, Rev. Mod. Phys. 43, 297 (1971).

[9] M. Inokuti, Y. Itikawa and J. E. Turner, Rev. Mod. Phys. 50, 23 (1978).

[10] C. B. Opal, W. K. Peterson and E. C. Beaty, J. Chem. Phys. 55, 4100 (1971).

[11] M. B. Shah, D. S. Elliott and H. B. Gilbody, J. Phys. B, 20, 3501 (1987).

[12] Y. -K. Kim and M. Inokuti, Phys. Rev. A3, 665 (1971).

[13] B. H. Bransden, A. Dalgarno, T. L. John and M. J. Seaton, Proc. Phys. Soc. 71, 877 (1958).

[14] A. Dalgarno and G. W. Griffing, Proc. R. Soc. London A, 248, 415 (1958).

[15] M. R. H. Rudge, J. Phys. B, 8, 940 (1975).

[16] J. N. Das and M. R. H. Rudge, J. Phys. B, 9, L131 (1976).

[17] W. L. van Wyngaarden and H. R. J. Walters, J. Phys. B, 19, 929 (1986).

[18] W. E. Swartz, J. S. Nisbet and A. E. S. Green, J. Geophys. res. 76, 8425 (1971).

[19] W. Heitler, The Quantum Theory of Radiation, 3rd ed., Oxford University Press, London (1954).

[20] K. R. Lang, Astrophysical Formulae, 3rd ed., Springer, Berlin (1999).

[21] A. A. Zdziarski and R. Svensson, Astrophys. J. 344, 551 (1989).

[22] F. C. Jones, Phys. Rev. 167, 1159 (1968).

[23] M. Inokuti, Argonne National Laboratory Report No. ANL-6769, p7 (1963).

[24] J. W. Motz, H. A. Olsen and H. W. Koch, Rev. Mod. Phys. 41, 581 (1969).

[25] L. C. Maximon, J. Res. NBS 72(B), 79 (1968).

[26] P. V. C. Hough, Phys. Rev. 73, 266 (1948).

[27] R. Svensson and A. A. Zdziarski, Astrophys. J. 349, 415 (1990).

[28] R. J. Gould and G. P. Schreder, Phys. Rev. 155, 1404 (1967).

[29] F. A. Agaronyan, A. M. Atyan and A. N. Najapetyan, Astrophysics 19, 187 (1983).

[30] C. J. Crannell, G. Joyce, R. Ramaty and C. Werntz, Astrophys. J. 210, 582 (1976).

[31] A. Ore and J. L. Powell, Phys. Rev. 75, 1696 (1949).

[32] A. A. Zdziarski, Astrophys. J. 335, 786 (1988). 